\title{
A TOPOLOGICAL APPROACH TO A CLASS OF ONE-DIMENSIONAL KIRCHHOFF EQUATIONS
}

\author{
CHRISTOPHER S. GOODRICH \\ (Communicated by Wenxian Shen)
}

This paper is dedicated to the memory of Maddie Goodrich (16 March 2002-16 March 2020).

Abstract. We consider, for $q>1$, the one-dimensional Kirchhoff-type problem

$$
\begin{aligned}
-A\left(\int_{0}^{1}\left(u^{\prime}(s)\right)^{q} d s\right) u^{\prime \prime}(t) & =\lambda f(u(t)), t \in(0,1) \\
u(0) & =0 \\
u^{\prime}(1) & =0
\end{aligned}
$$

and demonstrate the existence of at least one positive solution to this problem. The main contribution is to show that by using a nonstandard order cone together with topological fixed point theory much weaker conditions than usual can be imposed on the coefficient function $A$.

\section{INTRODUCTION}

In this paper we consider a one-dimensional Kirchhoff-type problem with rightfocal boundary conditions - namely, the problem

$$
\begin{aligned}
-A\left(\int_{0}^{1}\left(u^{\prime}(s)\right)^{q} d s\right) u^{\prime \prime}(t) & =\lambda f(u(t)), t \in(0,1) \\
u(0) & =0 \\
u^{\prime}(1) & =0,
\end{aligned}
$$

where $\lambda>0, q>1, A:[0,+\infty) \rightarrow \mathbb{R}$ is continuous, and $f: \mathbb{R} \rightarrow[0,+\infty)$ is continuous. The main contribution of this article is to use a novel order cone together with an associated nonstandard open set to study the existence of at least one positive solution to problem (1.1). As explained in a moment this is different than most other studies, which tend to use either variational methods or more standard order cones. Due to our methodology we are able to weaken notably the conditions imposed on the coefficient function $A$. Note that the coefficient $A$ acts as a sort-of nonlocal coefficient since $u^{\prime}$ is not localized at a specific point. Nonlocal differential equations have been studied extensively in recent years - see, for example, Goodrich [22,23, Goodrich and Lizama 30,31, Graef and Webb 32, Infante [36], Infante and Pietramala [38, Jankowski [40, Karakostas [41, Webb and Infante [45, and Yang [48.

Received by the editors December 25, 2020, and, in revised form, February 23, 2021.

2020 Mathematics Subject Classification. Primary 34B08, 34B10, 34B18, 47H10, 47H30; Secondary 26D15, 35J25, 35J60.

(C)2021 by the author under Creative Commons Attribution-Noncommercial 3.0 License (CC BY NC 3.0) 
Kirchhoff-type problems similar to (1.1) (or the elliptic PDE analogue) have been extensively studied in the existing literature - some recent and relevant examples are papers by Afrouzi, Chung, and Shakeri [1, Alves and Corrêa [2], Azzouz and Bensedik [5], Boulaaras [11, Boulaaras and R. Guefaifia [13, Cao and Dai [16, Chung [17, Infante [35, 37, and Liu, Luo, and Dai [42. In addition, a problem similar to (1.1) in which $\left\|u^{\prime}\right\|_{L^{q}}^{q}$ is replaced by $\|u\|_{L^{q}}^{q}$, e.g.,

$$
-A\left(\int_{0}^{1}(u(s))^{q} d s\right) u^{\prime \prime}(t)=\lambda f(u(t)), t \in(0,1),
$$

has also been studied extensively - see, for example, papers by Aly [4, Alves and Covei [3, Bavaud [6, Biler, Krzywicki, and Nadzieja [7, Biler and Nadzieja 8, 9], Caglioti, Lions, Marchioro, and Pulvirenti [15], Corrêa [19], Corrêa, Menezes, and Ferreira [20, do Ó, Lorca, and Ubilla [21], Goodrich [29], Stańczy [43, Wang, Wang, and An [44, Yan and Ma [46], and Yan and Wang [47. Such problems occur frequently in applications such as the mean field equation - see [35, (1.2)].

In these sorts of studies it is very common to assume some combination of the following conditions regarding the coefficient function $A$ :

(1) $A(t)>0$ for all $t \geq 0$.

(2) $A$ either satisfies some sort of global growth condition and/or some sort of global monotonicity condition.

(3) $A$ is either sub- or super-linear at either 0 or $+\infty$.

Evidently, in general, condition (1) is especially important so that the differential equation does not degenerate. For example, in Afrouzi, Chung, and Shakeri [1] the equivalent of $A$ is assumed to be bounded between two positive numbers. Similarly, in both Alves and Corrêa [2] and Azzouz and Bensedik [5] the equivalent of $A$ is assumed to be bounded away from zero and, in some results, a monotone increasing function. Likewise in Boulaaras and Guefaifia [13] the nonlocal coefficients (i.e., the equivalent of $A$ in our problem) are assumed to be globally bounded, globally increasing, and bounded away from zero. Similarly, the very recent papers by Bouizem, Boulaaras, and Djebbar [10, Boulaaras, Bouizem, and Guefaifia [12, and Boulaaras, Guefaifia, Cherif, and Radwan [14] also impose these types of conditions in the PDEs analogue of (1.1). More specifically, in [10,12 the equivalent of the function $A$ is bounded away from zero and, in addition, a largeness condition is imposed on the quantity $\inf _{s \geq 0} A(s)$ - see [10, pp. 2466 and (M2)] and [12, p. 9196 and (M2)]. Likewise in [14] two nonlocal coefficients are considered as part of a system of nonlocal PDEs, and the nonlocal coefficients are bounded away from zero and increasing [14, (H1)]. Furthermore, with the exception of [29], which we further mention below, it is a similar matter with the other papers mentioned in the preceding paragraph - e.g., [3, Condition (2) on p. 1], [17, Conditions (M0), (3), (4)], [21, Condition (H1), p. 299], 35, Theorem 2.3], [43, Theorem 2.2], [44, Condition (H3), p. 2], 46, p. 1], and [47, Theorem 4.1, p. 84].

One can see, then, that these types of conditions are pervasive in the existing literature, and this is due to having no direct control over the quantity $\int_{0}^{1}\left(u^{\prime}(s)\right)^{q} d s$, which thus requires one to make global assumptions about the behavior of the coefficient function $A$. Thus, by way of contrast we propose to study problem (1.1) by a topological approach using a nonstandard order cone. Recently the author [29] proposed a new way to treat problem (1.2) by restricting attention to those 
functions for which $u \mapsto\|u\|_{L^{1}}$ is coercive. This methodology completely avoided the use of conditions (1)-(3).

Inspired to take a similar approach we restrict attention to those functions $u \in$ $\mathscr{C}^{1}([0,1])$ such that, for a constant $C_{0} \in(0,1)$ defined in Section 2 ,

$$
u(1)=\int_{0}^{1} u^{\prime}(s) d s \geq C_{0}\left\|u^{\prime}\right\|_{\infty},
$$

so that the functional $u \mapsto \int_{0}^{1} u^{\prime}(s) d s$ is coercive; equivalently, this condition can be seen as a Harnack-like inequality. Together with the set

$$
\widehat{W}_{\rho}:=\left\{u \in \mathscr{K}: \int_{0}^{1}\left(u^{\prime}(s)\right)^{q} d s<\rho\right\}
$$

where $\mathscr{K}$ is a suitable positive cone defined in Section 2, this simple restriction turns out to be very useful because it allows us to characterize pointwise information regarding the argument of $A$. Indeed, we are able to deduce information about the exact value of $\int_{0}^{1}\left(u^{\prime}(s)\right)^{q} d s$ - the key topological fact being that when $u \in \partial \widehat{W}_{\rho}$ it follows that

$$
\int_{0}^{1}\left(u^{\prime}(s)\right)^{q} d s=\rho .
$$

Consequently, instead of having to assume some combination of (1)-(3) above, we assume none of this. Rather, we need only impose on condition on $A$ at two points - see condition (H2) in Section 2. Moreover, $A$ can be nonpositive on (possibly infinitely many) intervals of positive measure. It should be mentioned that although the methodology in the Kirchhoff case is inspired by 29], there are significant differences and a straightforward adaptation of the methodology in [29] fails. For example, here we must make careful use of reverse Hölder inequalities in order to suitably control certain quantities; this sort of complication does not arise at all in 29 .

All in all, we are able to eliminate (1)-(3) with no substantial increase in the restrictions imposed on the forcing term $f$. This is the advantage of using the set $\widehat{W}_{\rho}$. Essentially it affords us very precise control over the argument of the coefficient function $A$ in problem (1.1) in a way that both the variational methods and the standard order cones fail to do. Sets similar to $\widehat{W}_{\rho}$ have been previously used by the author in studying other types of nonlocal equation - see, for example, 24 27. But to the best of our knowledge they have never been used in the case of Kirchhoff-type equations, in which, as we have already mentioned, there are some extra difficulties due to the presence of $u^{\prime}$ in the nonlocal element.

Finally, while we have elected to study problem (1.1) in the case of right focal boundary conditions, we believe that the methods introduced in this paper can be adapted to other types of boundary data. In addition, other forcing terms can be accommodated, we believe, with minimal alteration in the essential methodology. Rather, we focus on the specific problem (1.1) in order to illustrate more clearly the methodology. 


\section{EXISTENCE RESUlT}

Let $G:[0,1] \times[0,1] \rightarrow[0,+\infty)$ be defined by

$$
G(t, s):=\left\{\begin{array}{ll}
t, & 0 \leq t \leq s \leq 1 \\
s, & 0 \leq s \leq t \leq 1
\end{array},\right.
$$

which is the Green's function for the right-focal problem $-u^{\prime \prime}(t)=0, t \in(0,1)$, subject to $u(0)=0$ and $u^{\prime}(1)=0$. Equip the space $\mathscr{C}^{1}([0,1])$ with the norm

$$
\|u\|:=\max \left\{\|u\|_{\infty},\left\|u^{\prime}\right\|_{\infty}\right\} \text {. }
$$

In what follows the function $G_{t}:([0,1] \times[0,1]) \backslash\{(t, s) \in[0,1] \times[0,1]: t=s\} \rightarrow \mathbb{R}$ denotes the function

$$
G_{t}(t, s):=\frac{\partial}{\partial t} G(t, s) \equiv\left\{\begin{array}{ll}
1, & 0 \leq t<s \leq 1 \\
0, & 0 \leq s<t \leq 1
\end{array} .\right.
$$

We note that although $G_{t}$ does not exist along the diagonal $\mathscr{D}:=\{(t, s) \in[0,1] \times$ $[0,1]: t=s\}$, this is of no consequence in what follows. Then denoting by $\mathscr{G}_{t}$ the function $\mathscr{G}_{t}(s):=\sup _{t \in[0,1] \backslash \mathscr{D}}\left|G_{t}(t, s)\right|$, it follows for a.e. $s \in[0,1]$ that $\mathscr{G}_{t}(s):=\sup _{t \in[0,1] \backslash \mathscr{D}}\left|G_{t}(t, s)\right|=1$.

Henceforth we will assume the following conditions on the functions $A$ and $f$ appearing in problem (1.1). Note that in condition (H2) we use for a continuous function $f:[a, b] \rightarrow \mathbb{R}$, where $0 \leq a<b<+\infty$, the notation

- $f_{[a, b]}^{m}:=\min _{y \in[a, b]} f(y)$; and

- $f_{[a, b]}^{M}:=\max _{y \in[a, b]} f(y)$.

We also use this notation in the proof of Theorem 2.9.

(H1): The function $f: \mathbb{R} \rightarrow[0, \infty)$ is continuous and satisfies the following conditions.

(1) $f(0) \neq 0$

(2) There exist numbers $C_{0} \in(0,1]$ and $\rho_{2}>0$ such that

(a) $f$ is increasing on the interval $\left[0, \frac{\rho_{2}^{\frac{1}{q}}}{C_{0}}\right]$;

(b) $C_{0}<\frac{f(0)}{f\left(\frac{\rho_{2}^{\frac{1}{q}}}{C_{0}}\right)} e^{-1}$.

(H2): Letting $\rho_{2}$ be as in condition (H1), the function $A:[0, \infty) \rightarrow \mathbb{R}$ is continuous, and, in addition, there exists a number $\rho_{1}>0$, with $0<\rho_{1}<$ $\rho_{2}$, such that

(1) $A(t)>0$ for each $t \in\left[\rho_{1}, \rho_{2}\right]$;

(2) $\left.f_{\left[0, \frac{\rho_{1}^{\frac{1}{q}}}{C_{0}}\right]}^{m}\right]^{-1}\left((q+1) \rho_{1}\right)^{\frac{1}{q}} A\left(\rho_{1}\right)$; and

(3)

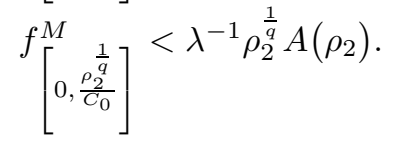

Remark 2.1. As mentioned in Section 1 the conditions imposed on $f$ via condition (H1) are not substantively worse (or better) than those in the existing literature. 
In particular, condition $(\mathrm{H} 1)(1)$ implies that $\lim _{x \rightarrow 0^{+}} \frac{f(x)}{x}=+\infty$. It is worth noting that if $f$ satisfies standard growth, i.e., $c_{1}\left(1+|u|^{p}\right) \leq f(u) \leq c_{2}\left(1+|u|^{p}\right)$ for $0<c_{1}<c_{2}$ and $p>1$, then condition (H1) can be satisfied, and standard growth is a physically relevant model. On the other hand and as explained more thoroughly in Section 1, the conditions imposed on $A$ via (H2) are much weaker than the standard ones in the literature. So, the upshot of the methodology here is not so much the conditions on the forcing term $f$ but rather the conditions on the nonlocal coefficient $A$.

Remark 2.2. Note that since $f(0) \neq 0$ and $f$ is increasing on $\left[0, \frac{\rho_{2}^{\frac{1}{q}}}{C_{0}}\right]$ it thus follows that $f\left(\frac{\rho_{2}^{\frac{1}{q}}}{C_{0}}\right) \neq 0$.

In the use of the topological fixed point theory we will work within the cone $\mathscr{K} \subseteq \mathscr{C}^{1}([0,1])$ defined by

$$
\mathscr{K}:=\left\{u \in \mathscr{C}^{1}([0,1]): u \geq 0, u^{\prime} \geq 0, \int_{0}^{1} u^{\prime}(s) d s \geq C_{0}\left\|u^{\prime}\right\|_{\infty}, u(0)=0\right\},
$$

where $C_{0} \in(0,1)$ satisfies the implicit functional inequality in condition $(\mathrm{H} 1)(2)(\mathrm{c})$ - namely,

$$
C_{0}<\frac{f(0)}{f\left(\frac{\rho_{2}^{\frac{1}{2}}}{C_{0}}\right)} .
$$

Importantly, we note that $C_{0}$ depends only on initial data - i.e., $C_{0}:=C_{0}\left(f ; \rho_{2}\right)$. Therefore, it can be calculated in a given problem - see Example 2.11.

Remark 2.3. Since $\int_{0}^{1} u^{\prime}(s) d s=u(1)-u(0)=u(1)$, one may rightly inquire as to why we use the integral formulation in the definition of $\mathscr{K}$. The reason is twofold. First, we wish to emphasize the connection to the integral of $u^{\prime}$ since this will be of central important in what follows. Second, it also possibly points the way toward treating the problem in the case of more general nonlocal coefficients or equipped with more general boundary conditions.

Attendant to the cone $\mathscr{K}$ define the open set $\widehat{W}_{\rho} \subseteq \mathscr{K}$ by

$$
\widehat{W}_{\rho}:=\left\{u \in \mathscr{K}: \int_{0}^{1}\left(u^{\prime}(s)\right)^{q} d s<\rho\right\} .
$$

Then

$$
\partial \widehat{W}_{\rho}:=\left\{u \in \mathscr{K}: \int_{0}^{1}\left(u^{\prime}(s)\right) d s=\rho\right\} .
$$

Finally, in what follows we will be concerned with the operator $T: \mathscr{K} \rightarrow \mathscr{C}^{1}([0,1])$ defined by

$$
(T u)(t):=\lambda \int_{0}^{1}\left(A\left(\int_{0}^{1}\left(u^{\prime}(r)\right)^{q} d r\right)\right)^{-1} G(t, s) f(u(s)) d s .
$$

A fixed point of $T$ is a solution of problem (1.1).

Prior to stating and proving our existence theorem we need to establish some preliminary lemmata. Our first lemma establishes a relationship between elements of the boundary set $\partial \widehat{W}_{\rho}$ and their norm in the ambient $\mathscr{C}^{1}$ norm $\|\cdot\|$. 
Lemma 2.4. Suppose that $u \in \partial \widehat{W}_{\rho}$. Define the set $\Omega_{\rho}:=\{u \in \mathscr{K}:\|u\|<\rho\}$. Then

$$
u \in \overline{\Omega_{\frac{\rho}{\rho^{\frac{1}{q}}}}} \backslash \Omega_{\rho^{\frac{1}{q}}} .
$$

Proof. Note that if $u \in \partial \widehat{W}_{\rho}$, then since $\|u\|:=\max \left\{\|u\|_{\infty},\left\|u^{\prime}\right\|_{\infty}\right\}$ it follows that

$$
\rho=\int_{0}^{1}\left(u^{\prime}(s)\right)^{q} d s \leq\left\|u^{\prime}\right\|_{\infty}^{q} \leq\|u\|^{q} .
$$

Therefore, $u \in \mathscr{K} \backslash \Omega_{\rho^{\frac{1}{q}}}$. At the same time, if $u \in \partial \widehat{W}_{\rho}$, then an application of Jensen's inequality (recall that $q>1$ ) yields the inequality

$$
\rho=\int_{0}^{1}\left(u^{\prime}(s)\right)^{q} d s \geq\left(\int_{0}^{1} u^{\prime}(s) d s\right)^{q} \geq C_{0}^{q}\left\|u^{\prime}\right\|_{\infty}^{q},
$$

where, since $u \in \mathscr{K}$, we have used the coercivity of the functional $u \mapsto \int_{0}^{1} u^{\prime}(s) d s$. Therefore, we conclude that

$$
\left\|u^{\prime}\right\|_{\infty} \leq \frac{\rho^{\frac{1}{q}}}{C_{0}}
$$

But then since $u \in \mathscr{K}$, it follows that $u(0)=0$ and $u \in \mathscr{C}^{1}([0,1])$. Thus, from inequality (2.1) we deduce that

$$
\|u\|_{\infty}:=\max _{t \in[0,1]}|u(t)| \leq \frac{\rho^{\frac{1}{q}}}{C_{0}} .
$$

Putting inequalities (2.1) $-(2.2)$ together yields $\|u\| \leq \frac{\rho^{\frac{1}{q}}}{C_{0}}$ so that $u \in \overline{\Omega_{\frac{\rho}{\rho_{0}}}}$. There-

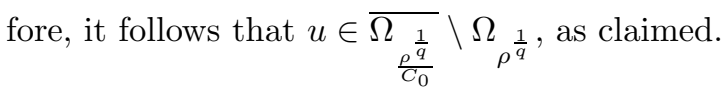

Remark 2.5. Note that one consequence of Lemma 2.4 is that the set $\partial \widehat{W}_{\rho}$ can be identified as a subset of a solid annular region with respect to the ambient norm $\|\cdot\|$.

The next lemma establishes the openness and boundedness of the set $\widehat{W}_{\rho}$ for each $\rho>0$. This is an essential property in order to use the topological fixed point theory.

Lemma 2.6. For each $\rho>0$ the set $\widehat{W}_{\rho}$ is both bounded and (relatively) open in $\mathscr{K}$.

Proof. The boundedness essentially follows from the proof of Lemma 2.4, Indeed, if $u \in \widehat{W}_{\rho}$, then

$$
\rho>\int_{0}^{1}\left(u^{\prime}(s)\right)^{q} d s \geq\left(\int_{0}^{1} u^{\prime}(s) d s\right)^{q} \geq C_{0}^{q}\left\|u^{\prime}\right\|_{\infty}^{q},
$$

where we have used Jensen's inequality. So, $\left\|u^{\prime}\right\|_{\infty} \leq \frac{\rho^{\frac{1}{q}}}{C_{0}}<+\infty$. Thus,

$$
\max _{t \in[0,1]}|u(t)|=\|u\|_{\infty} \leq \frac{\rho^{\frac{1}{q}}}{C_{0}}<+\infty .
$$


Therefore, $\|u\|=\max \left\{\|u\|_{\infty},\left\|u^{\prime}\right\|_{\infty}\right\}<+\infty$. By the arbitrariness of both $u \in \widehat{W}_{\rho}$ and $\rho>0$, we deduce that $\widehat{W}_{\rho}$ is bounded, as claimed. Finally, the openness of $\widehat{W}_{\rho}$, for each $\rho>0$, is a consequence of the definition of $\widehat{W}_{\rho}$ - i.e., the nonstrict inequality together with the fact that $\mathscr{K} \subseteq \mathscr{C}^{1}(\mathbb{R})$. And this completes the proof.

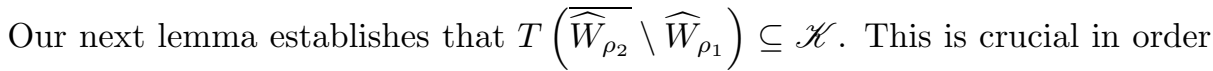
to obtain the fixed point result later. Note that we only require the reflexivity of

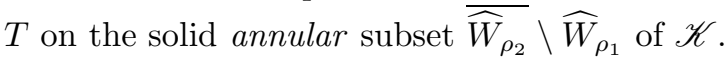

Lemma 2.7. Assume that each of conditions (H1) and (H2) is satisfied. Then

$$
T\left(\overline{\widehat{W}_{\rho_{2}}} \backslash \widehat{W}_{\rho_{1}}\right) \subseteq \mathscr{K}
$$

Proof. Our strategy to complete the proof is to use an argument similar to that used in [28, Lemma 2.8]. We will prove that for each sector $\partial \widehat{W}_{\rho}$, where $\rho_{1} \leq \rho \leq \rho_{2}$, it

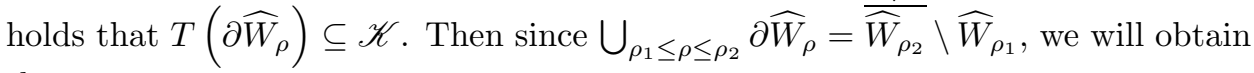
that

$$
T\left(\bigcup_{\rho_{1} \leq \rho \leq \rho_{2}} \partial \widehat{W}_{\rho}\right)=T\left(\overline{\widehat{W}_{\rho_{2}}} \backslash \widehat{W}_{\rho_{1}}\right) \subseteq \mathscr{K}
$$

Let us first note that

$$
\left(A\left(\int_{0}^{1}\left(u^{\prime}(r)\right)^{q} d r\right)\right)^{-1}>0
$$

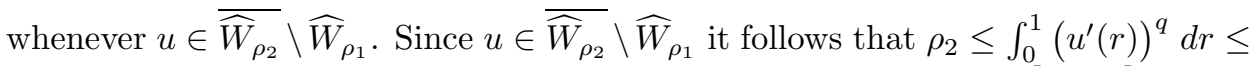
$\rho_{2}$. But from condition (H2) we know that $A(t)>0$ whenever $t \in\left[\rho_{1}, \rho_{2}\right]$. Thus, we see at once that the desired claim holds.

We next show that

$$
\int_{0}^{1}(T u)^{\prime}(t) d t \geq C_{0}\left\|(T u)^{\prime}\right\|_{\infty}
$$

So, fix a $\rho \in\left[\rho_{1}, \rho_{2}\right]$ and let $u \in \partial \widehat{W}_{\rho}$ be fixed but otherwise arbitrary. Now, recalling the formula for $(t, s) \mapsto G_{t}(t, s)$, we see that

$$
(T u)^{\prime}(t)=\lambda \int_{t}^{1}\left(A\left(\int_{0}^{1}\left(u^{\prime}(r)\right)^{q} d r\right)\right)^{-1} f(u(s)) d s
$$


because $G_{t}(t, s) \equiv 1$ when $s \in(t, 1)$ and $G_{t}(t, s) \equiv 0$ when $s \in(0, t)$ for each $t \in[0,1]$. So, in light of (2.4) we see that

$$
\begin{aligned}
\int_{0}^{1}(T u)^{\prime}(t) d t & =\lambda \int_{0}^{1}\left[\int_{0}^{1}\left(A\left(\int_{0}^{1}\left(u^{\prime}(r)\right)^{q} d r\right)\right)^{-1} G_{t}(t, s) f(u(s)) d s\right] d t \\
& =\lambda \int_{0}^{1}\left[\int_{t}^{1}\left(A\left(\int_{0}^{1}\left(u^{\prime}(r)\right)^{q} d r\right)\right)^{-1} f(u(s)) d s\right] d t \\
& =\lambda \int_{0}^{1} \int_{0}^{s}\left(A\left(\int_{0}^{1}\left(u^{\prime}(r)\right)^{q} d r\right)\right)^{-1} f(u(s)) d t d s \\
& =\lambda \int_{0}^{1}\left[\int_{0}^{s} d t\right]\left(A\left(\int_{0}^{1}\left(u^{\prime}(r)\right)^{q} d r\right)\right)^{-1} f(u(s)) d s \\
& =\lambda \int_{0}^{1}\left(A\left(\int_{0}^{1}\left(u^{\prime}(r)\right)^{q} d r\right)\right)^{-1} s f(u(s)) d s
\end{aligned}
$$

where we have used Fubini's theorem in order to interchange the order of integration in (2.5). On the other hand, keeping in mind that $\mathscr{G}_{t}(s)=1$ a.e., we also calculate

$$
\begin{aligned}
\left\|(T u)^{\prime}\right\|_{\infty} & \leq \lambda \int_{0}^{1}\left(A\left(\int_{0}^{1}\left(u^{\prime}(r)\right)^{q} d r\right)\right)^{-1} \mathscr{G}_{t}(s) f(u(s)) d s \\
& =\lambda \int_{0}^{1}\left(A\left(\int_{0}^{1}\left(u^{\prime}(r)\right)^{q} d r\right)\right)^{-1} f(u(s)) d s .
\end{aligned}
$$

Comparing inequalities (2.5) and (2.6) we see that the desired coercivity inequality (2.3) will hold provided that

$$
\begin{aligned}
0 & <C_{0} \\
& \leq \int_{0}^{1}\left(A\left(\int_{0}^{1}\left(u^{\prime}(r)\right)^{q} d r\right)\right)^{-1} s f(u(s)) d s \\
& / \int_{0}^{1}\left(A\left(\int_{0}^{1}\left(u^{\prime}(r)\right)^{q} d r\right)\right)^{-1} f(u(s)) d s \\
& =\int_{0}^{1} s f(u(s)) d s / \int_{0}^{1} f(u(s)) d s .
\end{aligned}
$$

We claim that the choice of $C_{0}$ defined implicitly by the relation

$$
C_{0}<\frac{f(0)}{f\left(\frac{\rho_{2}^{\frac{1}{2}}}{C_{0}}\right)} e^{-1},
$$

as assumed in condition (H1)(b), satisfies requirement (2.7) and, thus, completes the proof.

To see that this claim is true note that inequality (2.8) implies the existence of an integer $n>2$ sufficiently large such that

$$
C_{0} \leq\left(\frac{n-1}{n-2}\right)^{1-n} \frac{f(0)}{f\left(\frac{\rho_{2}^{\frac{1}{2}}}{C_{0}}\right)}
$$


since $\left(\frac{n-1}{n-2}\right)^{1-n}>0$ for each $n>2, n \mapsto\left(\frac{n-1}{n-2}\right)^{1-n}$ is increasing for $n>2$, and

$$
\lim _{n \rightarrow \infty}\left(\frac{n-1}{n-2}\right)^{1-n}=\frac{1}{e} .
$$

Then for such $n$ chosen sufficiently large we note that the reverse Hölder inequality implies that

$$
\begin{aligned}
\int_{0}^{1} s f(u(s)) d s & \geq\left(\int_{0}^{1} s^{-\frac{1}{n-1}} d s\right)^{-n+1}\left(\int_{0}^{1}(f(u(s)))^{\frac{1}{n}} d s\right)^{n} \\
& =\left(\frac{n-1}{n-2}\right)^{1-n}\left(\int_{0}^{1}(f(u(s)))^{\frac{1}{n}} d s\right)^{n} \\
& \geq\left(\frac{n-1}{n-2}\right)^{1-n}\left(\int_{0}^{1}(f(0))^{\frac{1}{n}} d s\right)^{n} \\
& =\left(\frac{n-1}{n-2}\right)^{1-n} f(0),
\end{aligned}
$$

where we have used the monotonicity of $f$ via condition $(\mathrm{H} 1)(1)$ to write $f(u(s)) \geq$ $f(0)$ since $u$ is nonnegative by virtue of the fact that $u \in \mathscr{K}$. At the same time, using the monotonicity of $f$ again, together with the fact that $u(s) \leq u(1)$ since $u^{\prime}$ is nonnegative by virtue of the fact that $u \in \mathscr{K}$, we also deduce that

$$
\int_{0}^{1} f(u(s)) d s \leq f(u(1))
$$

Now recalling that $u \in \partial \widehat{W}_{\rho}$ we notice from Jensen's inequality that

$$
\rho=\int_{0}^{1}\left(u^{\prime}(s)\right)^{q} d s \geq\left(\int_{0}^{1} u^{\prime}(s) d s\right)^{q} \geq\left(C_{0}\left\|u^{\prime}\right\|_{\infty}\right)^{q}
$$

so that

And since $u(0)=0$ this means that

$$
\left\|u^{\prime}\right\|_{\infty} \leq \frac{\rho^{\frac{1}{q}}}{C_{0}} \leq \frac{\rho_{2}^{\frac{1}{q}}}{C_{0}}
$$

$$
u(1) \leq \frac{\rho_{2}^{\frac{1}{q}}}{C_{0}} .
$$

Therefore,

$$
\int_{0}^{1} f(u(s)) d s \leq f(u(1)) \leq f\left(\frac{\rho_{2}^{\frac{1}{q}}}{C_{0}}\right)
$$

using the monotonicity of $f$ once more.

Let us note that the monotonicity calculations in each of (2.10), (2.11), and (2.13) are valid since by assumption $(\mathrm{H} 1)(1)$, the function $f$ is increasing on $\left[0, \frac{\rho_{2}^{\frac{1}{q}}}{C_{0}}\right]$, and as inequality (2.12) demonstrates we have that

$$
0 \leq u(s) \leq u(1) \leq \frac{\rho_{2}^{\frac{1}{q}}}{C_{0}}
$$


Consequently, the monotonicity calculations are justifiable.

Therefore, putting inequalities (2.10) and (2.13) together we deduce that

$$
\int_{0}^{1} s f(u(s)) d s / \int_{0}^{1} f(u(s)) d s \geq\left(\frac{n-1}{n-2}\right)^{1-n} \frac{f(0)}{f\left(\frac{\rho_{2}^{\frac{1}{q}}}{C_{0}}\right)} \geq C_{0},
$$

where the final inequality follows from inequality (2.9) and the choice of $n$. Finally, putting inequalities (2.5)-(2.7) and (2.14) together we conclude that

$$
\begin{aligned}
\int_{0}^{1}(T u)^{\prime}(t) d t & =\lambda \int_{0}^{1}\left(A\left(\int_{0}^{1}\left(u^{\prime}(r)\right)^{q} d r\right)\right)^{-1} s f(u(s)) d s \\
& =\lambda\left(A\left(\int_{0}^{1}\left(u^{\prime}(r)\right)^{q} d r\right)\right)^{-1} \int_{0}^{1} s f(u(s)) d s \\
& \geq \lambda\left(A\left(\int_{0}^{1}\left(u^{\prime}(r)\right)^{q} d r\right)\right)^{-1} C_{0} \int_{0}^{1} f(u(s)) d s \\
& =C_{0} \lambda \int_{0}^{1}\left(A\left(\int_{0}^{1}\left(u^{\prime}(r)\right)^{q} d r\right)\right)^{-1} \mathscr{G}_{t}(s) f(u(s)) d s \\
& \geq C_{0}\left\|(T u)^{\prime}\right\|_{\infty},
\end{aligned}
$$

which establishes the desired coercivity inequality for $(T u)^{\prime}$ on $\partial \widehat{W}_{\rho}$. Moreover, due the arbitrariness of $\rho \in\left[\rho_{1}, \rho_{2}\right]$ the argument outlined in the first paragraph of the proof then proves that the coercivity condition is satisfied on the set $\widehat{W}_{\rho_{2}} \backslash \widehat{W}_{\rho_{1}}$, as desired.

Finally, that both $(T u)(t) \geq 0$ and $(T u)^{\prime}(t) \geq 0$, for each $t \in[0,1]$, is a consequence of the definition of each of these operators. Indeed, since both $G$ and $G_{t}$ are nonnegative along with $f$ and $A$, the subsequent nonnegativity of $T u$ and $(T u)^{\prime}$ follow at once. Furthermore, each of $T u$ and $(T u)^{\prime}$ is continuous. So, we conclude that $T\left({\widehat{W_{\rho_{2}}}}_{\widehat{W}_{\rho_{1}}}\right) \subseteq \mathscr{K}$, as claimed.

Our final lemma recalls a classical fixed point result. One may consult, for example, Cianciaruso, Infante, and Pietramala [18, Lemma 2.3], Guo and Lakshmikantham 34, Infante, Pietramala, and Tenuta [39], or Zeidler [49] for further details on this and similar results.

Lemma 2.8. Let $U$ be a bounded open set and, with $\mathcal{K}$ a cone in a real Banach space $\mathscr{X}$, suppose both that $U_{\mathscr{K}}:=U \cap \mathcal{K} \supseteq\{0\}$ and that $\overline{U_{\mathscr{K}}} \neq \mathscr{K}$. Assume that $T: \overline{U_{\mathscr{K}}} \rightarrow \mathscr{K}$ is a compact map such that $x \neq T x$ for each $x \in \partial U_{\mathscr{K}}$. Then the fixed point index $i_{\mathscr{K}}\left(T, U_{\mathscr{K}}\right)$ has the following properties.

(1) If there exists $e \in \mathscr{K} \backslash\{0\}$ such that $x \neq T x+\lambda e$ for each $x \in \partial U_{\mathscr{K}}$ and each $\lambda>0$, then $i_{\mathscr{K}}\left(T, U_{\mathscr{K}}\right)=0$.

(2) If $\mu x \neq T x$ for each $x \in \partial U_{\mathscr{K}}$ and for each $\mu \geq 1$, then $i_{\mathscr{K}}\left(T, U_{\mathscr{K}}\right)=1$.

(3) If $i_{\mathscr{K}}\left(T, U_{\mathscr{K}}\right) \neq 0$, then $T$ has a fixed point in $U_{\mathscr{K}}$.

(4) Let $U^{1}$ be open in $X$ with $\overline{U_{\mathscr{K}}^{1}} \subseteq U_{\mathscr{K}}$. If $i_{\mathscr{K}}\left(T, U_{\mathscr{K}}\right)=1$ and $i_{\mathscr{K}}\left(T, U_{\mathscr{K}}^{1}\right)=$ 0 , then $T$ has a fixed point in $U_{\mathscr{K}} \backslash \overline{U_{\mathscr{K}}^{1}}$. The same result holds if $i_{\mathscr{K}}\left(T, U_{\mathscr{K}}\right)$ $=0$ and $i_{\mathscr{K}}\left(T, U_{\mathscr{K}}^{1}\right)=1$. 
With the preliminary lemmata dispatched we are now able to state and prove the existence result for problem (1.1).

Theorem 2.9. Assume that conditions (H1)-(H2) are satisfied. Then problem (1.1) has at least one positive solution, $u_{0}$, satisfying the localization

$$
u_{0} \in \Omega_{\frac{\rho_{2}^{\frac{1}{q}}}{C_{0}}} \backslash \overline{\Omega_{\rho_{1}^{\frac{1}{q}}}} .
$$

Proof. We first demonstrate that for each $\mu>0$ and $u \in \partial \widehat{W}_{\rho_{1}}$ it holds that $u \neq T u+\mu e$, where $e(t):=t$. Note that $e \in \mathscr{K}$ since $e(t) \geq 0, e^{\prime}(t) \equiv 1 \geq 0$, $e(0)=0$, and $\int_{0}^{1} e^{\prime}(t) d t=1 \geq C_{0}=C_{0}\left\|e^{\prime}\right\|_{\infty}$.

So, suppose for contradiction that $u=T u+\mu e$ for some $u \in \partial \widehat{W}_{\rho_{1}}$ and $\mu>0$. Then $u^{\prime}(t)=(T u)^{\prime}(t)+\mu e^{\prime}(t)=(T u)^{\prime}(t)+\mu>(T u)^{\prime}(t)$ for each $t \in[0,1]$. Recalling that

$$
\int_{0}^{1}\left(u^{\prime}(t)\right)^{q} d t=\rho_{1}
$$

since $u \in \partial \widehat{W}_{\rho_{1}}$, it follows upon integrating both sides of $\left(u^{\prime}\right)^{q}(t)>\left((T u)^{\prime}\right)^{q}(t)$ from $t=0$ to $t=1$ that

$$
\begin{aligned}
\rho_{1} & =\int_{0}^{1}\left(u^{\prime}(t)\right)^{q} d t \\
& >\int_{0}^{1}\left((T u)^{\prime}(t)\right)^{q} d t \\
& =\int_{0}^{1} \underbrace{\left[\lambda \int_{0}^{1}\left(A\left(\int_{0}^{1}\left(u^{\prime}(r)\right)^{q} d r\right)\right)^{-1} G_{t}(t, s) f(u(s)) d s\right]^{q} d t}_{=\left((T u)^{\prime}(t)\right)^{q}} \\
& =\int_{0}^{1}\left[\lambda \int_{0}^{1}\left(A\left(\rho_{1}\right)\right)^{-1} G_{t}(t, s) f(u(s)) d s\right]^{q} d t \\
& \geq \frac{\lambda^{q}}{\left(A\left(\rho_{1}\right)\right)^{q}} \int_{0}^{1}\left(\int_{t}^{1} f(u(s)) d s\right)^{q} d t \\
& \geq \frac{\lambda^{q}}{\left(A\left(\rho_{1}\right)\right)^{q}}\left(f_{\left[0, \frac{\rho_{1}^{q}}{C_{0}}\right.}^{m}\right)^{q} \int_{0}^{1}(1-t)^{q} d t \\
& =\frac{\lambda^{q}}{(q+1)\left(A\left(\rho_{1}\right)\right)^{q}}\left[f_{\left[0, \frac{\rho_{1}^{\frac{1}{q}}}{C_{0}}\right]}^{m}\right)^{q},
\end{aligned}
$$

which by condition $(\mathrm{H} 2)(2)$ is a contradiction. Note that in (2.17) we have used Lemma 2.4 to assert that $\left.f(u(s)) \leq f_{[}^{m}{ }^{\frac{1}{q}}\right]$ for each $s \in[0,1]$. Therefore, we conclude by Lemma 2.8 that

$$
\left[0, \frac{\rho_{1}^{\frac{1}{q}}}{C_{0}}\right]
$$

$$
i_{\mathscr{K}}\left(T, \widehat{W}_{\rho_{1}}\right)=0
$$


On the other hand, we claim that $\mu u \neq T u$ for each $u \in \partial \widehat{W}_{\rho_{2}}$ and for each $\mu \geq 1$. Instead suppose for contradiction that $\mu u(t)=(T u)(t)$ for each $t \in[0,1]$ and some $u \in \partial \widehat{W}_{\rho_{2}}$ and $\mu \geq 1$. Then $\mu u^{\prime}(t)=(T u)^{\prime}(t)$, whereupon taking both sides of this equality to the $q$-th power and integrating from $t=0$ to $t=1$ we obtain in a manner similar to (2.17) that

$$
\begin{aligned}
\rho_{2} \leq \mu^{q} \int_{0}^{1}\left(u^{\prime}(t)\right)^{q} d t & =\int_{0}^{1}\left((T u)^{\prime}(t)\right)^{q} d t \\
& =\int_{0}^{1}\left[\lambda \int_{0}^{1}\left(A\left(\rho_{2}\right)\right)^{-1} G_{t}(t, s) f(u(s)) d s\right]^{q} d t \\
& \leq \frac{\lambda^{q}}{\left(A\left(\rho_{2}\right)\right)^{q}}\left(f_{\left[\begin{array}{c}
M \\
{\left[0, \frac{\rho_{2}}{C_{0}}\right.}
\end{array}\right]}^{q},\right.
\end{aligned}
$$

where we used equality (2.16) with $\rho_{2}$ replacing $\rho_{1}$. Since (2.19) is by (H2)(3) a contradiction, we conclude from Lemma 2.8 that

$$
i_{\mathscr{K}}\left(T, \widehat{W}_{\rho_{2}}\right)=1 \text {. }
$$

Finally, upon combining (2.18) and (2.20) we deduce from Lemma 2.8 the existence of $u_{0} \in \widehat{W}_{\rho_{2}} \backslash{\widehat{W_{\rho_{1}}}}$ such that $T u_{0}=u_{0}$. Therefore, from the proof of Lemma 2.4 we deduce that

$$
\rho_{1}^{\frac{1}{q}}<\|u\|<\frac{\rho_{2}^{\frac{1}{q}}}{C_{0}}
$$

so that $u_{0}$ satisfies the localization in the statement of the theorem. Finally, since $u_{0}$ is also a positive solution of (1.1), the proof of the theorem is complete.

Remark 2.10. We point out that although the statement of Lemma 2.8 appears to require that $T$ map the entirety of the set $\overline{U_{\mathscr{K}}}$ into $\mathscr{K}$, it is sufficient for $T$ to map the solid annular region $\overline{U_{\mathscr{K}}} \backslash U_{\mathscr{K}}^{1}$ into $\mathscr{K}$ - just as we have used in the proof of Theorem 2.9. One can argue this by using Dugundji's extension theorem [33, Theorem 2.5].

We conclude with a simple example in order to illustrate the application of Theorem 2.9.

Example 2.11. For $1.22473<\lambda<1.49565$ consider the problem

$$
-10 \cos \left(\int_{0}^{1}\left(u^{\prime}(s)\right)^{2} d s\right) u^{\prime \prime}(t)=\lambda\left((u(t))^{\frac{1}{2}}+1\right), t \in(0,1)
$$

subject to the boundary data $u(0)=0=u^{\prime}(1)$. From (2.21) we identify that $q=2$, $A(t):=10 \cos t$, and $f(u):=\sqrt{u}+1$. Clearly, (H1)(1) and (H1)(2)(a) are satisfied for any $\rho_{2}$ and $C_{0}$. If we choose $C_{0}:=\frac{3}{25}$, then condition (H1)(2)(b) leads to the inequality $\rho_{2} \lesssim 0.26218$. Choose $\rho_{1}:=0.005$ and $\rho_{2}:=0.2$. Then condition (H2)(1) is satisfied since $10 \cos t>0$ for $t \in[0.005,0.2]$, condition $(\mathrm{H} 2)(2)$ is satisfied since it reduces to $1>\frac{10}{\lambda} \sqrt{3 \rho_{1}} \cos \rho_{1}$, and condition $(\mathrm{H} 2)(3)$ is satisfied since it reduces to

$$
\sqrt{\frac{\sqrt{\rho_{2}}}{C_{0}}}+1<\frac{10}{\lambda} \sqrt{\rho_{2}} \cos \rho_{2}
$$


Furthermore, it can be shown that (to five decimal places of accuracy) for each $1.22473<\lambda<1.49565$ these inequalities are satisfied. Therefore, Theorem 2.9 implies the existence of at least one positive solution, $u_{0}$, to problem (2.21) satisfying the localization

$$
0.071 \approx \sqrt{\frac{1}{200}}<\max \left\{\left\|u_{0}\right\|_{\infty},\left\|u_{0}^{\prime}\right\|_{\infty}\right\}<\frac{25}{3} \sqrt{\frac{1}{5}} \approx 3.727 .
$$

Remark 2.12. Notice that the coefficient function, $A(t)=10 \cos t$, in Example 2.11 is negative on infinitely many intervals of positive measure. Moreover, it is zero infinitely often. Consequently, as described in Section 1 we do not believe that nonlocal equation (2.21) could be treated by the existing methods in the literature.

\section{REFERENCES}

[1] G. A. Afrouzi, N. T. Chung, and S. Shakeri, Existence and non-existence results for nonlocal elliptic systems via sub-supersolution method, Funkcial. Ekvac. 59 (2016), no. 3, 303-313, DOI 10.1619/fesi.59.303. MR.3642538

[2] Claudianor O. Alves and Francisco Julio S. A. Corrêa, A sub-supersolution approach for a quasilinear Kirchhoff equation, J. Math. Phys. 56 (2015), no. 5, 051501, 12, DOI 10.1063/1.4919670. MR.3390975

[3] Claudianor O. Alves and Dragoş-Pătru Covei, Existence of solution for a class of nonlocal elliptic problem via sub-supersolution method, Nonlinear Anal. Real World Appl. 23 (2015), 1-8, DOI 10.1016/j.nonrwa.2014.11.003. MR.3316619

[4] J. J. Aly, Thermodynamics of a two-dimensional self-gravitating system, Phys. Rev. E (3) 49 (1994), no. 5, 3771-3783, DOI 10.1103/PhysRevE.49.3771. MR1380234

[5] N. Azzouz and A. Bensedik, Existence results for an elliptic equation of Kirchhoff-type with changing sign data, Funkcial. Ekvac. 55 (2012), no. 1, 55-66, DOI 10.1619/fesi.55.55. MR.2976042

[6] François Bavaud, Equilibrium properties of the Vlasov functional: the generalized PoissonBoltzmann-Emden equation, Rev. Modern Phys. 63 (1991), no. 1, 129-148, DOI 10.1103/RevModPhys.63.129. MR:1102194

[7] Piotr Biler, Andrzej Krzywicki, and Tadeusz Nadzieja, Self-interaction of Brownian particles coupled with thermodynamic processes, Rep. Math. Phys. 42 (1998), no. 3, 359-372, DOI 10.1016/S0034-4877(99)80005-6. MR.1674322

[8] Piotr Biler and Tadeusz Nadzieja, A class of nonlocal parabolic problems occurring in statistical mechanics, Colloq. Math. 66 (1993), no. 1, 131-145, DOI 10.4064/cm-66-1-131-145. MR 1242651

[9] Piotr Biler and Tadeusz Nadzieja, Nonlocal parabolic problems in statistical mechanics, Proceedings of the Second World Congress of Nonlinear Analysts, Part 8 (Athens, 1996), Nonlinear Anal. 30 (1997), no. 8, 5343-5350, DOI 10.1016/S0362-546X(96)00165-4. MR1726036

[10] Youcef Bouizem, Salah Boulaaras, and Bachir Djebbar, Some existence results for an elliptic equation of Kirchhoff-type with changing sign data and a logarithmic nonlinearity, Math. Methods Appl. Sci. 42 (2019), no. 7, 2465-2474, DOI 10.1002/mma.5523. MR.3936413

[11] Salah Boulaaras, Existence of positive solutions for a new class of Kirchhoff parabolic systems, Rocky Mountain J. Math. 50 (2020), no. 2, 445-454, DOI 10.1216/rmj.2020.50.445. MR 4104385

[12] Salah Boulaaras, Youcef Bouizem, and Rafik Guefaifia, Further results of existence of positive solutions of elliptic Kirchhoff equation with general nonlinearity of source terms, Math. Methods Appl. Sci. 43 (2020), no. 15, 9195-9205, DOI 10.1002/mma.6613. MR4151400

[13] Salah Boulaaras and Rafik Guefaifia, Existence of positive weak solutions for a class of Kirrchoff elliptic systems with multiple parameters, Math. Methods Appl. Sci. 41 (2018), no. 13, 5203-5210, DOI 10.1002/mma.5071. MR.3843588

[14] Salah Boulaaras, Rafik Guefaifia, Bahri Cherif, and Taha Radwan, Existence result for a Kirchhoff elliptic system involving p-Laplacian operator with variable parameters and additive right hand side via sub and super solution methods, AIMS Math. 6 (2021), no. 3, 2315-2329, DOI 10.3934/math.2021140. MR4199165 
[15] E. Caglioti, P.-L. Lions, C. Marchioro, and M. Pulvirenti, A special class of stationary flows for two-dimensional Euler equations: a statistical mechanics description, Comm. Math. Phys. 143 (1992), no. 3, 501-525. MR1145596

[16] Xiaofei Cao and Guowei Dai, Spectrum global bifurcation and nodal solutions to Kirchhofftype equations, Electron. J. Differential Equations (2018), Paper No. 179, 10. MR.3891484

[17] Nguyen Thanh Chung, Existence of positive solutions for a class of Kirchhoff type systems involving critical exponents, Filomat 33 (2019), no. 1, 267-280, DOI 10.2298/fil1901267c. MR3940073

[18] Filomena Cianciaruso, Gennaro Infante, and Paolamaria Pietramala, Non-zero radial solutions for elliptic systems with coupled functional BCs in exterior domains, Proc. Edinb. Math. Soc. (2) 62 (2019), no. 3, 747-769, DOI 10.1017/s0013091518000706. MR.3974965

[19] F. J. S. A. Corrêa, On positive solutions of nonlocal and nonvariational elliptic problems, Nonlinear Anal. 59 (2004), no. 7, 1147-1155, DOI 10.1016/j.na.2004.08.010. MR2098510

[20] F. J. S. A. Corrêa, Silvano D. B. Menezes, and J. Ferreira, On a class of problems involving a nonlocal operator, Appl. Math. Comput. 147 (2004), no. 2, 475-489, DOI 10.1016/S00963003(02)00740-3. MR2012587

[21] João Marcos do Ó, Sebastián Lorca, Justino Sánchez, and Pedro Ubilla, Positive solutions for some nonlocal and nonvariational elliptic systems, Complex Var. Elliptic Equ. 61 (2016), no. 3, 297-314, DOI 10.1080/17476933.2015.1064404. MR.3454108

[22] C. S. Goodrich, On nonlocal BVPs with nonlinear boundary conditions with asymptotically sublinear or superlinear growth, Math. Nachr. 285 (2012), no. 11-12, 1404-1421, DOI 10.1002/mana.201100210. MR2959967

[23] Christopher S. Goodrich, On nonlinear boundary conditions involving decomposable linear functionals, Proc. Edinb. Math. Soc. (2) 58 (2015), no. 2, 421-439, DOI 10.1017/S0013091514000108. MR3341447

[24] Christopher S. Goodrich, The effect of a nonstandard cone on existence theorem applicability in nonlocal boundary value problems, J. Fixed Point Theory Appl. 19 (2017), no. 4, 26292646, DOI 10.1007/s11784-017-0448-7. MR3720473

[25] Christopher S. Goodrich, Coercive nonlocal elements in fractional differential equations, Positivity 21 (2017), no. 1, 377-394, DOI 10.1007/s11117-016-0427-z. MR3613003

[26] Christopher S. Goodrich, New Harnack inequalities and existence theorems for radially symmetric solutions of elliptic PDEs with sign changing or vanishing Green's function, J. Differential Equations 264 (2018), no. 1, 236-262, DOI 10.1016/j.jde.2017.09.011. MR3712941

[27] Christopher S. Goodrich, Radially symmetric solutions of elliptic PDEs with uniformly negative weight, Ann. Mat. Pura Appl. (4) 197 (2018), no. 5, 1585-1611, DOI 10.1007/s10231018-0738-8. MR 3848465

[28] Christopher S. Goodrich, Perturbed integral operator equations of Volterra type with applications to p-Laplacian equations, Mediterr. J. Math. 15 (2018), no. 2, Paper No. 47, 20, DOI 10.1007/s00009-018-1090-3. MR 3769712

[29] C. S. Goodrich, A topological approach to nonlocal elliptic partial differential equations on an annulus, Math. Nachr. 294 (2021), 286-309.

[30] Christopher Goodrich and Carlos Lizama, Positivity, monotonicity, and convexity for convolution operators, Discrete Contin. Dyn. Syst. 40 (2020), no. 8, 4961-4983, DOI 10.3934/dcds.2020207. MR4112036

[31] C. S. Goodrich, C. Lizama, Existence and monotonicity of nonlocal boundary value problems: the one-dimensional case, Proc. Roy. Soc. Edinburgh Sect. A, doi: 10.1017/prm.2020.90.

[32] John R. Graef and J. R. L. Webb, Third order boundary value problems with nonlocal boundary conditions, Nonlinear Anal. 71 (2009), no. 5-6, 1542-1551, DOI 10.1016/j.na.2008.12.047. MR2524369

[33] Andrzej Granas and James Dugundji, Fixed point theory, Springer Monographs in Mathematics, Springer-Verlag, New York, 2003. MR.1987179

[34] Da Jun Guo and V. Lakshmikantham, Nonlinear problems in abstract cones, Notes and Reports in Mathematics in Science and Engineering, vol. 5, Academic Press, Inc., Boston, MA, 1988. MR959889

[35] Gennaro Infante, Nonzero positive solutions of nonlocal elliptic systems with functional BCs, J. Elliptic Parabol. Equ. 5 (2019), no. 2, 493-505, DOI 10.1007/s41808-019-00049-6. MR 4031965 
[36] Gennaro Infante, Positive and increasing solutions of perturbed Hammerstein integral equations with derivative dependence, Discrete Contin. Dyn. Syst. Ser. B 25 (2020), no. 2, 691-699, DOI 10.3934/dcdsb.2019261. MR4043586

[37] G. Infante, Eigenvalues of elliptic functional differential systems via a Birkhoff-Kellogg type theorem, Mathematics 9 (2021), No. 1:4.

[38] Gennaro Infante and Paolamaria Pietramala, Nonzero radial solutions for a class of elliptic systems with nonlocal BCs on annular domains, NoDEA Nonlinear Differential Equations Appl. 22 (2015), no. 4, 979-1003, DOI 10.1007/s00030-015-0311-8. MR.3385628

[39] Gennaro Infante, Paolamaria Pietramala, and Mattia Tenuta, Existence and localization of positive solutions for a nonlocal BVP arising in chemical reactor theory, Commun. Nonlinear Sci. Numer. Simul. 19 (2014), no. 7, 2245-2251, DOI 10.1016/j.cnsns.2013.11.009. MR3157933

[40] Tadeusz Jankowski, Positive solutions to fractional differential equations involving Stieltjes integral conditions, Appl. Math. Comput. 241 (2014), 200-213, DOI 10.1016/j.amc.2014.04.080. MR.3223422

[41] George L. Karakostas, Existence of solutions for an $n$-dimensional operator equation and applications to BVPs, Electron. J. Differential Equations (2014), No. 71, 17. MR3193977

[42] Fang Liu, Hua Luo, and Guowei Dai, Global bifurcation and nodal solutions for homogeneous Kirchhoff type equations, Electron. J. Qual. Theory Differ. Equ., posted on 2020, Paper No. 29, 13, DOI 10.14232/ejqtde.2020.1.29. MR 4098341

[43] Robert Stańczy, Nonlocal elliptic equations, Proceedings of the Third World Congress of Nonlinear Analysts, Part 5 (Catania, 2000), Nonlinear Anal. 47 (2001), no. 5, 3579-3584, DOI 10.1016/S0362-546X(01)00478-3. MR.1979257

[44] Yunhai Wang, Fanglei Wang, and Yukun An, Existence and multiplicity of positive solutions for a nonlocal differential equation, Bound. Value Probl., posted on 2011, 2011:5, 11, DOI 10.1186/1687-2770-2011-5. MR2821484

[45] J. R. L. Webb and Gennaro Infante, Positive solutions of nonlocal boundary value problems: a unified approach, J. London Math. Soc. (2) 74 (2006), no. 3, 673-693, DOI 10.1112/S0024610706023179. MR2286439

[46] Baoqiang Yan and Tianfu Ma, The existence and multiplicity of positive solutions for a class of nonlocal elliptic problems, Bound. Value Probl., posted on 2016, Paper No. 165, 35, DOI 10.1186/s13661-016-0670-z. MR3546370

[47] Baoqiang Yan and Dechen Wang, The multiplicity of positive solutions for a class of nonlocal elliptic problem, J. Math. Anal. Appl. 442 (2016), no. 1, 72-102, DOI 10.1016/j.jmaa.2016.04.023. MR3498319

[48] Zhilin Yang, Existence and nonexistence results for positive solutions of an integral boundary value problem, Nonlinear Anal. 65 (2006), no. 8, 1489-1511, DOI 10.1016/j.na.2005.10.025. MR2246350

[49] Eberhard Zeidler, Nonlinear functional analysis and its applications. I, Springer-Verlag, New York, 1986. Fixed-point theorems; Translated from the German by Peter R. Wadsack. MR 816732

School of Mathematics and Statistics, UNSW Sydney, Sydney, NSW 2052 Australia

Email address: c.goodrich@unsw.edu.au 